\title{
BMJ Open Awake prone positioning of hypoxaemic patients with COVID-19: protocol for a randomised controlled open-label superiority meta-trial
}

\author{
Elsa Tavernier (D) , ${ }^{1,2}$ Bairbre McNicholas, ${ }^{3,4}$ Ivan Pavlov, ${ }^{5}$ Oriol Roca, ${ }^{6,7}$ \\ Yonatan Perez, ${ }^{8}$ John Laffey, ${ }^{3,4}$ Sara Mirza, ${ }^{9}$ David Cosgrave, ${ }^{3,4}$ David Vines, ${ }^{9}$ \\ Jean-Pierre Frat, ${ }^{10}$ Stephan Ehrmann, ${ }^{8} \mathrm{Jie} \mathrm{Li} \mathrm{(i)}{ }^{9}$
}

To cite: Tavernier E,

McNicholas B, Pavlov I, et al. Awake prone positioning of hypoxaemic patients with COVID-19: protocol for a randomised controlled open-label superiority meta-trial. BMJ Open 2020;10:e041520. doi:10.1136/ bmjopen-2020-041520

- Prepublication history and supplemental material for this paper is available online. To view these files, please visit the journal online (http://dx.doi org/10.1136/bmjopen-2020041520).

Received 11 June 2020 Revised 08 September 2020 Accepted 06 October 2020
D) Check for updates

(C) Author(s) (or their employer(s)) 2020. Re-use permitted under CC BY-NC. No commercial re-use. See rights and permissions. Published by BMJ.

For numbered affiliations see end of article.

Correspondence to

Dr Elsa Tavernier;

elsa.tavernier@univ-tours.fr

\section{ABSTRACT}

Introduction Prone positioning (PP) is an effective firstline intervention to treat patients with moderate to severe acute respiratory distress syndrome (ARDS) receiving invasive mechanical ventilation, as it improves gas exchanges and reduces mortality. The use of PP in awake spontaneous breathing patients with ARDS secondary to COVID-19 was reported to improve oxygenation in few retrospective trials with small sample size. High-level evidence of awake PP for hypoxaemic patients with COVID-19 patients is still lacking.

Methods and analysis The protocol of this meta-trial is a prospective collaborative individual participant data meta-analysis of randomised controlled open label superiority trials. This design is particularly adapted to a rapid scientific response in the pandemic setting. It will take place in multiple sites, among others in USA, Canada, Ireland, France and Spain. Patients will be followed up for 28 days. Patients will be randomised to receive whether awake PP and nasal high flow therapy or standard medical treatment and nasal high flow therapy. Primary outcome is defined as the occurrence rate of tracheal intubation or death up to day 28. An interim analysis plan has been set up on aggregated data from the participating research groups.

Ethics and dissemination Ethics approvals were obtained in all participating countries. Results of the metatrial will be submitted for publication in a peer-reviewed journal. Each randomised controlled trial was registered individually, as follows: NCT04325906, NCT04347941, NCT04358939, NCT04395144 and NCT04391140.

\section{INTRODUCTION}

\section{Background and rationale}

COVID-19 is an emerging infectious disease that was first reported in Wuhan, China, and had subsequently spread worldwide. As of 6 June 2020, more than 6 million cases were confirmed globally, and close to 0.4 million deaths were reported. ${ }^{1}$ Nearly $20 \%$ of patients experienced hypoxemia, which was the primary reason for hospitalisation. ${ }^{2}$ In patients with severe disease who were admitted to the intensive care unit
Strengths and limitations of this study

- This pragmatic design will deal with the recruitment difficulties that could occur in the individual trials given the uncertainties of the international dynamics of the COVID-19 pandemic.

- The collaborative interim analysis plan at the level of the meta-trial will enable an earlier data analysis compared with the individual study level or to a retrospective meta-analysis.

- Besides synthesising the effect size estimates, it also considers the aspect of replication: results being consistent across trials is a strength in favour of a robust treatment effect over different conditions.

- The lack of blinding of trial participants, care providers and outcome assessors is an unavoidable limitation of the study design.

(ICU), mortality rates of up to $42 \%$ have been described. $^{3}$ As of 6 June 2020, 51.2\% of the 6128 UK hospitalised patients with COVID-19 that required advanced respiratory support died $^{3}$ and $36 \%$ mortality was reported for invasively ventilated COVID-19 patients in a single centre in Atlanta. ${ }^{4}$

High flow nasal cannula (HFNC) oxygen therapy provides oxygen-rich heated humidified gas to the patient's nose at flow rates sufficient to deliver a constant, precisely set high fraction of inspired oxygen $\left(\mathrm{FiO}_{2}\right)$. HFNC washes out the dead space carbon dioxide, provides a low level of positive end-expiratory pressure and decreases breathing frequency and work of breathing. ${ }^{5}{ }^{6}$ In hypoxaemic respiratory failure, HFNC use is associated with lower mortality, lower rates on endotracheal intubation and improved oxygenation. ${ }^{7-9}$ It has been extensively used early in the COVID-19 outbreak in China. ${ }^{10}$

Prone positioning (PP) of mechanically ventilated patients is an effective first-line 
intervention to treat patients with moderate to severe acute respiratory distress syndrome (ARDS) receiving invasive mechanical ventilation, as it improves gas exchanges and reduces mortality. ${ }^{11}{ }^{12}$ There is limited evidence to support awake PP of patients treated with HFNC. Two small studies showed that PP was feasible in spontaneously breathing patients. ${ }^{13}{ }^{14}$ In one of them, PP combined with HFNC resulted in higher arterial partial pressure of oxygen $\left(\mathrm{PaO}_{2}\right)$ to $\mathrm{FiO}_{2}$ ratios than HFNC alone. ${ }^{13}$ However, not all hypoxaemic patients with COVID-19 responded to awake PP. ${ }^{15} \mathrm{In}$ a retrospective study of 610 patients from China, ${ }^{16}$ a multipronged intervention that included early and aggressive use of HFNC and non-invasive ventilation (NIV) along with PP for awake patients resulted in lower overall mortality (3.33\%, as compared with $4.34 \%$ in a nearby province). A very low percentage of patients required mechanical ventilation $\left(<1 \%\right.$, as compared with the national average of $2.3 \%,{ }^{17}$ in a population that included $10 \%$ of critically ill patients). The authors highlighted that mortality was lower than in a previously reported cohort study of patients with ARDS performed at the same institution prior to the pandemic, ${ }^{18}$ although is not clear if the two populations were comparable in terms of disease severity. Since the outbreak, the use of awake PP with different oxygen modalities has been described in case series reports by teams from the USA, France, Italy and China. ${ }^{19-23}$ However, none of them provided high-level evidence of the effects on patients' outcome.

Based on the potential beneficial mechanisms of HFNC and PP, early use of PP combined with HFNC to avoid the need for intubation in COVID-19 patients with moderate to severe ARDS needs to be further investigated.

Due to the urgent need to find effective treatments for COVID-19, this meta-trial will gather together several trials launched independently at the beginning of the COVID-19 pandemic. As of 6 May 2020, eight randomised trials evaluating the efficacy of PP in patients with COVID-19 were registered on ClinicalTrials.gov. Early in the pandemic, we organised a meeting with the investigators and methodologists of the teams whose trials planned to include similar populations to address the same question of the effects of PP. We have decided to combine our recruitment capabilities and design an international meta-trial. ${ }^{24} 25$ This protocol includes a common analysis plan for the primary endpoint with four interim analysis in order to obtain early evidence.

\section{Objectives}

The primary objective is to demonstrate the efficacy of PP combined with HFNC in terms of treatment failure rate at 28 days, defined as a combination of (1) death and (2) intubation, in awake and spontaneously breathing patients with suspected or confirmed COVID-19 infection.

\section{METHODS AND ANALYSIS}

\section{Trial design}

This meta-trial is designed as a collaborative individual participant prospective data meta-analysis of five randomised controlled open-label superiority trials with two parallel groups and a primary endpoint of therapeutic failure at day 28 .

\section{Study setting}

This meta-trial will include patients with severe COVID-19 pneumonia treated with HFNC in the ICU, in emergency departments, in high-dependency units and on medical wards of participating hospitals. A full list of participating institutions is available in each individual trial record on ClinicalTrials.gov. The original protocols are in online supplemental files 1-4).

\section{Eligibility criteria}

All adult patients with proven (or clinically suspected, pending microbiological confirmation) COVID-19 pneumonia who require treatment with HFNC are eligible for this trial.

Eligibility criteria for potential trial participants are described in table 1 .

\section{Recruitment}

Due to the rapidly evolving pandemic situation, we have a strong uncertainty about the pace of enrolment. We anticipate this international collaboration to lead to better recruitment than individual trials studying the same population. Other individual RCTs may be added into this meta-trial study, as long as inclusion criteria, main outcomes and trial interventions are sufficiently similar.

\section{Interventions}

\section{Control group}

The patients in the control groups will be treated according to the same standard of care and receive the same oxygenation support with HFNC as in the intervention groups, but they will not be asked to remain in prone position. Details for each trial are presented in table 2.

\section{Intervention description}

The patients in the intervention groups will turn in prone position with the help and under the supervision of a caregiver to ensure that they are predominantly on their chest rather than on their side. Patients will be asked to remain in prone position as long as they can and as close as possible to 16 hours or more per day or more.

\section{Criteria for continuing or modifying allocated interventions}

Proning procedure will continue as long as the patient is in the following oxygen conditions:

- $\mathrm{PaO}_{2} / \mathrm{FiO}_{2}$ below 200 or $\mathrm{SpO}_{2}$ (peripheral oximetry saturation) to $\mathrm{FiO}_{2}$ ratio below 235 in the Irish trial.

- $\mathrm{PaO}_{2} / \mathrm{FiO}_{2}$ (or $\mathrm{SpO}_{2} / \mathrm{FiO}_{2}$ ) below $300 \mathrm{~mm} \mathrm{Hg}$ (or $315)$ in the French and Spanish trials.

- $\mathrm{PaO}_{2} / \mathrm{FiO}_{2}$ below $200 \mathrm{~mm} \mathrm{Hg}$ or $\mathrm{SpO}_{2} / \mathrm{FiO}_{2}$ below 240 in the Canadian and American trial.

Proning will be left at the discretion of the clinician in case of intubation.

Proning will be interrupted in case of discharge or death. 
Table 1 Eligibility criteria in each trial

\begin{tabular}{|c|c|c|c|}
\hline & USA and Canada & Ireland & France and Spain \\
\hline Inclusion criteria & $\begin{array}{l}\text { 1. COVID-19 pneumonia based on the } \\
\text { Ccenters for Disease Control guidelines. } \\
\text { 2. Presence of acute hypoxaemic respiratory } \\
\text { failure. } \\
\text { 3. Acute onset within } 7 \text { days of insult or new } \\
\text { (within } 7 \text { days) or worsening respiratory } \\
\text { symptoms. } \\
\text { 4. Bilateral opacities on chest } \mathrm{X} \text {-ray or } \mathrm{CT} \\
\text { scanner not fully explained by effusions, } \\
\text { lobar or lung collapse, or nodules. } \\
\text { 5. Cardiac failure not the primary cause of } \\
\text { acute respiratory failure. } \\
\text { 6. Written informed consent } \\
\text { 7. PaO } \mathrm{PaO}_{2} / \mathrm{FiO} \text { ratio }<200 \mathrm{~mm} \mathrm{Hg} \text { or } \mathrm{SpO}_{2} / \mathrm{FiO}_{2} \\
<240 \text { with } \mathrm{HFNC} \text { at } 50 \mathrm{~L} / \mathrm{min} \text { and peripheral } \\
\text { capillary oxygen saturation }\left(\mathrm{SpO}_{2)}\right. \\
\text { maintained at } 92 \%-95 \% \text {. }\end{array}$ & $\begin{array}{l}\text { 1. Suspected or confirmed } \\
\text { COVID- } 19 \text { infection. } \\
\text { 2. Bilateral Infiltrates on chest } \\
\text { X-ray } \\
\mathrm{SpO}_{2}<94 \% \text { on } \mathrm{FiO} \mathrm{O}_{2} 40 \% \text { by ei- } \\
\text { ther venturi facemask or } \mathrm{HFNC} \\
\text { 3. Respiratory rate }<40 \text { breath } / \mathrm{min} \text {. } \\
\text { 4. Written informed consent. }\end{array}$ & $\begin{array}{l}\text { 1. Adult patient suffering from COVID-19 } \\
\text { pneumonia according to the diagnostic } \\
\text { criteria in effect at the time of inclusion or } \\
\text { very strongly suspected. } \\
\text { 2. Patient treated by nasal high flow therapy. } \\
\text { 3. Moderate or severe ARDS: bilateral } \\
\text { radiological opacities not explained entirely } \\
\text { by effusions, atelectasis or nodules; acute } \\
\text { hypoxaemia with worsening within the seven } \\
\text { previous days, not entirely explained by left } \\
\text { ventricular failure; } \mathrm{PaO}_{2} / \mathrm{FiO}_{2} \text { ratio }<300 \mathrm{~mm} \\
\mathrm{Hg} \text { (or equivalent } \mathrm{SpO}_{2} / \mathrm{FiO}_{2} \text { ). } \\
\text { 4. Written informed consent in France and oral } \\
\text { consent in Spain. }\end{array}$ \\
\hline
\end{tabular}

ARDS, acute respiratory distress syndrome; $\mathrm{FiO}_{2}$, fraction of inspired oxygen; $\mathrm{HFNC}$, high flow nasal cannula; $\mathrm{PaO}$, partial pressure of oxygen; PP, prone positioning.

The following guidance is provided concerning the need for tracheal intubation to perform invasive mechanical ventilation. Intubation is recommended in case of $\mathrm{f}^{7}$ :

1. Signs of persisting or worsening respiratory failure, defined by at least two of the following criteria:

- Respiratory rate above 40 breaths/min.
- Lack of improvement of signs of respiratory muscle fatigue.

- Development of copious tracheal secretions.

- Hypercapnic respiratory acidosis with a $\mathrm{pH}$ below 7.25 .

Table 2 Standard management in each trial

\begin{tabular}{|c|c|c|}
\hline USA and Canada & Ireland & France and Spain \\
\hline $\begin{array}{l}\text { HFNC will be initiated at } 50 \mathrm{~L} / \mathrm{min} \text { (AIRVO2 or } \\
\text { Optiflow, Fisher \& Paykel Healthcare Limited, } \\
\text { Auckland, New Zealand) with temperature set } \\
\text { at } 37^{\circ} \mathrm{C} \text {. Nasal cannula size will be determined } \\
\text { by the patient's nostril size }(\leq 50 \%) \text {. FiO }{ }_{2} \text { will be } \\
\text { adjusted to maintain } \mathrm{SpO}_{2} \text { at } 92 \%-95 \% \text {. Flow } \\
\text { and temperature will be adjusted based on } \\
\text { patient's comfort and clinical response. }\end{array}$ & $\begin{array}{l}\text { Control patients will receive full } \\
\text { standard care. }\end{array}$ & $\begin{array}{l}\mathrm{HFNC} \text { adapted for an } \mathrm{SpO}_{2} \text { of } 90 \%-95 \% \text {. } \\
\text { Except in case of poor tolerance by the patient } \\
\text { a minimum gas flow rate of } 50 \mathrm{~L} / \mathrm{min} \text { will be } \\
\text { set initially. Weaning of the } \mathrm{HFNC} \text { will first be } \\
\text { performed reducing } \mathrm{FiO}_{2} \text { down to } 0.4 \text { before } \\
\text { reducing the gas flow rate. In clinically stable } \\
\text { patients with a } \mathrm{FiO}_{2} \text { less than or equal to } 0.4 \\
\text { and a gas flow rate less than or equal to } 30 \mathrm{~L} / \\
\text { min, an attempt will be made to switch to } \\
\text { standard oxygen therapy at } 4-6 \mathrm{~L} / \mathrm{min} \text {. }\end{array}$ \\
\hline
\end{tabular}

$\mathrm{FiO}_{2}$, fraction of inspired oxygen; HFNC, high flow nasal cannula. 
- $\mathrm{SpO}_{2}$ below $90 \%$ at $\mathrm{FiO}_{2} \geq 0.8$ for more than $5 \mathrm{~min}$ without technical dysfunction.

2. Haemodynamic instability.

3. Deterioration of neurological status.

For patients who meet the intubation criteria in the HFNC and HFNC+PP groups, a trial of NIV might be allowed according to the physician's preference in patients with signs of persisting or worsening respiratory failure and no other organ dysfunction before performing endotracheal intubation and invasive ventilation. Reasons for intubation will be recorded as well.

\section{Strategies to improve adherence to interventions}

The number of sessions and the total time spent in prone position will be collected per 24-hour period, and encouragement will be provided.

\section{Relevant concomitant care permitted or prohibited during the trial}

No prohibitions during the trial.

\section{Provisions for post-trial care}

Post-trial care will be standard care through the standard healthcare system from each country.

\section{Outcomes}

The primary outcome is therapeutic failure within 28 days of randomisation, defined as intubation (successful or attempted) or death.

\section{Secondary outcomes}

- Days spent in the ICU and in the hospital (within 28 days of randomisation).

- Mortality in the ICU and in the hospital (within 28 days of randomisation).

- Primary outcome (intubation of death) among patients receiving NIV in each randomisation groups.

- Time of escalation of therapy (in case of NIV use).

- Length of HFNC therapy use in those patients who succeeded with HFNC (efficacy).

- Length of HFNC therapy in those patients who fail with HFNC (safety).

- Ventilator-free days within the first 28 days.

- Need for rescue treatments in those patients who need to be intubated.

- Need for tracheotomy.

- Organ failure different from respiratory failure.

- Number of protocol violations.

- Time to intubation or death.

- Response to prone position: prechange and postchange of $\mathrm{SpO}_{2} / \mathrm{FiO}_{2}$ ratio, respiratory rate and ROX index $\left(\mathrm{SpO}_{2} /\left(\mathrm{FiO}_{2} \times\right.\right.$ respiratory rate $)$. As a practical alternative to $\mathrm{PaO}_{2} / \mathrm{FiO}_{2}, \mathrm{SpO}_{2} / \mathrm{FiO}_{2}$ has been shown to have a strong linear relationship in moderate to severe ARDS. ${ }^{26} 27$

- Duration of participation will be limited to 28 days after randomisation for each patient.

- Daily duration with PP in the first 3 days after enrolment.
Association between time of onset and outcome.

\section{Other measures}

In the PP groups complications will be recorded; complications include skin breakdown, device removal or desaturation during position change (within 28 days of randomisation).

\section{Plans for assessment and collection of outcomes}

Protocol explanation will be provident to study sites during a dedicated online or physical meeting. Assessment and collection of outcomes will be performed by investigators, physicians, nurses and research assistants trained and used to deal with patients with hypoxaemia without additional training required. $\mathrm{SpO}_{2} / \mathrm{FiO}_{2}$ ratio assessment requires the $\mathrm{SpO}_{2}$ to be equal of less than $97 \%$. The primary outcome (intubation or death) is easily retrieved from patients' charts. Bedside sheets are made available to simplify data recording. Each individual study coordinator is responsible for data quality control.

\section{Statistical methods}

\section{Sample size}

We assume the primary outcome rate to be between $60 \%$ and $70 \%$ in the control group. The meta-analysis is designed to demonstrate superiority of PP over control with $90 \%$ power and a one-sided type I error rate of 2.5. For a fixed design with no interim analysis and a sample size of 836 , the maximum detectable risk ratio will be between 0.847 and 0.814 (a difference of failure rates of about $11 \%$ between groups). For the same assumptions, asymmetric two-sided group sequential analysis requires a sample size of 1000, for five interim analyses (including the last analysis). Bounds were determined using a KimDeMets spending function with parameters 0.75 for efficacy and 3 for futility. This provides an aggressive Pocock superiority bound and a conservative O'Brien Fleming bound for futility (figure 1). Sample sizes were computed using the packages epiR and gsDesign in R software.

\section{Randomisation}

All patients who give consent for participation and who fulfil the inclusion criteria will be randomised. For each trial, a professional statistician not involved in patient recruitment will generate the allocation sequence. Participants will be randomly assigned to either control or experimental group with a 1:1 allocation as per a computer-generated randomisation schedule stratified by site and using varying block sizes. The American trial will also be stratified by ARDS severity (moderate vs severe), and French and Spanish trial will also be stratified by the therapeutic use of the PP prior to inclusion. In four trials, participants will be randomised using an online central randomisation system. In the Canadian trial, allocation concealment will be ensured using on-site sealed opaque envelopes. By the very nature of the interventions and design, trial participants, care providers, outcome assessors and data analysts could not be blinded to interventions. 


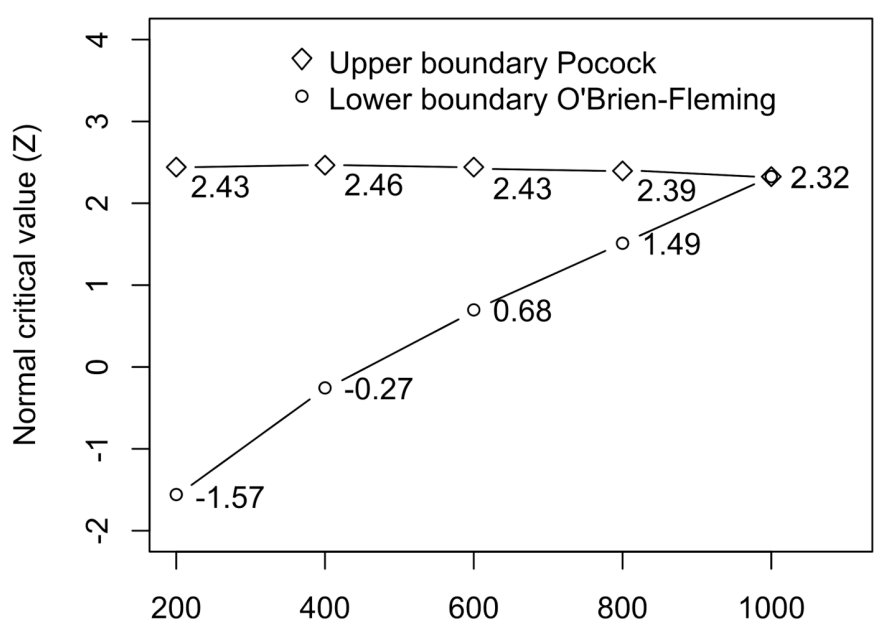

Sample size

Figure 1 Efficacy and futility stopping boundaries: analyses are planned every 200 patients randomised in the various trials. The interim analyses define rules for stopping the trials early for the statistical reasons of established efficacy or futility on the primary outcome. Bounds were determined using a Kim-DeMets spending function with an aggressive Pocock superiority and a conservative O'Brien Fleming bound for futility.

\section{Statistical methods for primary and secondary outcomes}

We plan a prospective meta-analysis of individual data. Common variables from all datasets will be gathered and combined to conduct the analysis. A detailed analysis plan will be a priori defined. The primary analysis will be performed on an intent-to-treat basis. A sensitivity analysis will be performed on a per-protocol set described below. Baseline patient characteristics will be presented by country and treatment group. The comparison between intervention arms will be synthesised using mixed-effects models with a random effect on the trial: a mixed-effects logistic regression for the primary outcome and any binary outcome. A survival analysis will be performed on mortality and any other time-to-event outcome, using a gamma-frailty term on each trial in a Cox regression model providing that the assumption of proportional hazards is verified. Regarding adverse events, descriptive statistics (percentages) will be estimated. We plan to assess statistical heterogeneity between countries by visual inspection of the forest plots, which will also present percountry analyses, and by calculating the $Q$ and $I^{2}$ statistics.

\section{Interim analyses}

We chose a Kim-DeMets alpha-spending approach ${ }^{28} 29$ rather than other methods such as a triangular test for its simplicity of implementation and for the continuous stopping boundaries enabling to be more flexible in managing interim analysis if the design of the trial were to change as a result of an unexpected development of the epidemic.

Analyses are planned when the total number of randomised patients with the primary outcome available from the various trials reaches 200 (100 in each arm),
400 (200 in each arm), 600 (300 in each arm), 800 (400 in each arm) and 1000 the last possible analysis. The interim analyses define rules for stopping the trials early for the statistical reasons of established efficacy or futility on the primary outcome. Two professional academic statisticians will conduct all interim analyses (blind duplicates).

At each interim analysis, the $\mathrm{Z}$ statistics for a difference of binary endpoints is computed from the data of the two arms and is compared with the efficacy and futility bounds given in figure 1 .

If the value of $\mathrm{Z}$ is higher than the interim analysis specific upper bound (or lower than the lower bound), the trials will be considered to be stopped for reasons of demonstrated efficacy (or futility), and data will be published as soon as possible to inform the clinical and scientific community; otherwise the trials will continue.

Methods for additional analyses (eg, subgroup analyses)

We plan to conduct a subgroup analysis on the severity of ARDS: $\mathrm{PaO}_{2} / \mathrm{FiO}_{2}$ ratio below $150 \mathrm{~mm} \mathrm{Hg}, \mathrm{PaO}_{2} / \mathrm{FiO}_{2}$ ratio above $150 \mathrm{~mm} \mathrm{Hg}$ (or equivalent $\mathrm{SpO}_{2} / \mathrm{FiO}_{2}$ ratio). We will test if the treatment effects differ with severity of ARDS by putting their main effect and interaction terms in the logistic regression.

Adjusted analyses will be nested in the intervention group to evaluate the effect of duration of $\mathrm{PP}$ on the risk of intubation or death, as well as the analysis of prognostic factors associated with PP such as comorbidities, age, body mass index and so on.

\section{Methods in analysis to handle protocol non-adherence and any} statistical methods to handle missing data

We do not expect any patient to be lost to follow-up. The only missing data could relate to patients who withdraw their consent. In this case, we will perform multiple imputations on the primary outcome. We will analyse the primary outcome using two analysis sets: the intention-totreat set, considering all patients as randomised regardless of whether they performed the prone position, and the per-protocol analysis set. The per-protocol set will only include patients who spent at least 1 hour in prone position after randomisation without intubation or death. Patients in the intervention group who spent less than 1 hour daily in PP and patients in the control group who remained more than 1 hour at least 1 day in PP will be excluded.

\section{ETHICS AND DISSEMINATION}

\section{Ethics and consent}

Ethics approval was obtained in all five participating countries. Informed consent will be obtained according to local regulations in each trial. Local investigators will obtain either verbal or electronic consent. Documentation of consent will be either written or electronic. 


\section{Data management, transfer and deposition}

The details of data management procedures can be found in the original protocols (online supplemental files). Each investigator is responsible for the confidentiality of the data collected during his or her trial. The data sets will use pseudonymised data. Interim analyses will be performed by centralising the aggregated data of the primary endpoint per trial. The confidentiality of data will be preserved when the coded, depersonalised data will be transmitted and stored at the location of the statistician in charge of the final analysis.

\section{Steering committee}

The steering committee will be responsible for reporting and interpreting the result of the interim analysis and the final analysis. The steering committee will be composed of principal investigators and statistician from all sites and may be completed by independent investigators without any competing interest. This study will be reported in accordance with the Consolidated Standards of Reporting Trials statement for non-pharmacological trials and published in peer-reviewed journals.

\section{Dissemination strategy}

The results of the study will be presented in national and international conferences and published via a peerreviewed journal.

\section{Data sharing statement}

Deidentified data will be made available on reasonable request discussed among the steering committee.

\section{Study status}

At the time of submitting for publication, the study was collecting data.

\section{Author affiliations}

${ }^{1}$ Clinical Investigation Center, INSERM 1415, CHRU Tours, Tours, Centre, France

${ }^{2}$ Methods in Patients-Centered Outcomes and Health Research, INSERM UMR 1246,

Nantes, Loire-Atlantique, France

${ }^{3}$ Department of Anaesthesia and Intensive Care Medicine, School of Medicine,

National University of Ireland Galway, Galway, Ireland

${ }^{4}$ Department of Anaesthesia, University Hospital Galway, Saolta University Hospital Group, Galway, Ireland

${ }^{5}$ Department of Emergency Medicine, Hopital de Verdun, Montreal, Quebec, Canada

${ }^{6}$ Critical Care Department, Vall d'Hebron University Hospital, Vall d'Hebron Research Institute, Barcelona, Catalunya, Spain

${ }^{7}$ Departament de Medicina, Universitat Autònoma de Barcelona, Barcelona, Catalunya, Spain

${ }^{8}$ Médecin Intensive Réanimation, CIC 1415, CRICS-TriggerSEP, Centre d'étude des pathologies respiratoires, INSERM U1100, Université de Tours, CHU Tours, Tours, Centre, France

${ }^{9}$ Department of Cardiopulmonary Sciences, Division of Respiratory Care, Rush

University, Chicago, Illinois, USA

${ }^{10}$ Réanimation Médicale, CHU Poitiers, Poitiers, France

\section{Twitter David Cosgrave @drdavidc9}

Acknowledgements The trial is supported by the following international clinical research networks: Réseau europpéen de recherche en ventilation artificielle (REVA: www.reseau-reva.org) and Clinical research in intensive care and sepsis - Trial group for global evaluation and research in sepsis (CRICS-TriggerSEP: www.triggersep.orgwww.triggersep.org endorsed by the French clinical research infrastructure network: www.fcrin.org).
Contributors BM, IP, OR, YP, JoL, SM, DC, J-PF, SE and JiL conceived the trials. ET provided methodological expertise in the meta-trial design and prepared the first draft of this study protocol. BM, IP, OR, YP, JoL, SM, DC, J-PF, SE and JiL contributed to the rewriting; all of the authors approved the final manuscript and reviewed it for important intellectual content.

Funding This work was supported by OpenAl. (3180 18th St, San Francisco, CA 94110, USA; no grant number), Rice Foundation (8600 Gross Point Rd, Skokie, IL 60077, USA; no grant number), Fisher \& Paykel (15 Maurice Paykel Place, East Tamaki, Auckland 2013 New Zealand; grant number P0 480003859) and the French ministry of health (14 Avenue Duquesne, 75350 Paris, France; grant number API20/T001).

Competing interests IP has been a speaker for Fisher \& Pakyel Healthcare within the last 3 years. All compensation was paid to the charitable foundation at the hospital where he works, and he did not personally receive any compensation. JL discloses research support from Fisher \& Paykel Healthcare for another research project. SE received unrestricted research grants, travel fee reimbursements and speaker fees from Fisher \& Paykel Healthcare, consulting fees from La Diffusion Technique Française, consulting fees and unrestricted research grants from Aerogen Ltd and an unrestricted research grant from Hamiton medical. OR provides consultancy to Hamilton Medical but he did not receive any personal fee. All compensation were received by the Institute of Research of his Institution. He received speaker fees by Air Liquide. J-PF reports grants from the French Ministry of Health; grants, personal fees and non-financial support from Fisher \& Paykel Heathcare; personal fees and non-financial support from SOS oxygene, outside the submitted work.

Patient consent for publication Not required.

Provenance and peer review Not commissioned; externally peer reviewed.

Supplemental material This content has been supplied by the author(s). It has not been vetted by BMJ Publishing Group Limited (BMJ) and may not have been peer-reviewed. Any opinions or recommendations discussed are solely those of the author(s) and are not endorsed by BMJ. BMJ disclaims all liability and responsibility arising from any reliance placed on the content. Where the content includes any translated material, BMJ does not warrant the accuracy and reliability of the translations (including but not limited to local regulations, clinical guidelines, terminology, drug names and drug dosages), and is not responsible for any error and/or omissions arising from translation and adaptation or otherwise.

Open access This is an open access article distributed in accordance with the Creative Commons Attribution Non Commercial (CC BY-NC 4.0) license, which permits others to distribute, remix, adapt, build upon this work non-commercially, and license their derivative works on different terms, provided the original work is properly cited, appropriate credit is given, any changes made indicated, and the use is non-commercial. See: http://creativecommons.org/licenses/by-nc/4.0/.

ORCID iDs

Elsa Tavernier http://orcid.org/0000-0003-0798-1182

Jie Li http://orcid.org/0000-0003-0121-1291

\section{REFERENCES}

1 WHO. Coronavirus disease (COVID-19) Dashboard. Available: https:// covid19.who.int/ [Accessed 26 May 2020].

2 Wu Z, McGoogan JM. Characteristics of and Important Lessons From the Coronavirus Disease 2019 (COVID-19) Outbreak in China: Summary of a Report of 72314 Cases From the Chinese Center for Disease Control and Prevention. JAMA 2020;323:1239-42.

3 ICNARC. ICNARC report on COVID-19 in critical care, 2020. Available: https://www.icnarc.org/DataServices/Attachments/ Download/66023c20-53a7-ea11-9126-00505601089b

4 Auld SC, Caridi-Scheible M, Blum JM, et al. ICU and ventilator mortality among critically ill adults with coronavirus disease. Crit Care Med 2019.

5 Nishimura M. High-Flow nasal cannula oxygen therapy in adults: physiological benefits, indication, clinical benefits, and adverse effects. Respir Care 2016;61:529-41.

6 Baker K, Greaves T, Fraser JF. How to use humidified high-flow nasal cannula in Breathless adults in the emergency department. Emerg Med Australas 2019;31:863-8.

7 Frat J-P, Thille AW, Mercat A, et al. High-Flow oxygen through nasal cannula in acute hypoxemic respiratory failure. $N$ Engl J Med 2015;372:2185-96. 
8 Rochwerg B, Granton D, Wang DX, et al. High flow nasal cannula compared with conventional oxygen therapy for acute hypoxemic respiratory failure: a systematic review and meta-analysis. Intensive Care Med 2019;45:563-72.

9 Li J, Jing G, Scott JB. Year in review 2019: high-flow nasal cannula oxygen therapy for adult subjects. Respir Care 2020;65:545-57.

10 Zhou F, Yu T, Du R, et al. Clinical course and risk factors for mortality of adult inpatients with COVID-19 in Wuhan, China: a retrospective cohort study. Lancet 2020;395:1054-62.

11 Guérin C, Reignier J, Richard J-C, et al. Prone positioning in severe acute respiratory distress syndrome. $N$ Engl J Med 2013;368:2159-68.

12 Scholten EL, Beitler JR, Prisk GK, et al. Treatment of ARDS with prone positioning. Chest 2017;151:215-24.

13 Ding L, Wang L, Ma W, et al. Efficacy and safety of early prone positioning combined with HFNC or NIV in moderate to severe ARDS: a multi-center prospective cohort study. Crit Care 2020;24:28.

14 Scaravilli V, Grasselli G, Castagna L, et al. Prone positioning improves oxygenation in spontaneously breathing nonintubated patients with hypoxemic acute respiratory failure: a retrospective study. J Crit Care 2015;30:1390-4.

15 Despres C, Brunin Y, Berthier F, et al. Prone positioning combined with high-flow nasal or conventional oxygen therapy in severe Covid-19 patients. Crit Care 2020;24:256.

16 Sun Q, Quu H, Huang M, et al. Lower mortality of COVID-19 by early recognition and intervention: experience from Jiangsu Province. Ann Intensive Care 2020;10:33

17 Guan W-J, Ni Z-Y, Hu Y, et al. Clinical characteristics of coronavirus disease 2019 in China. N Engl J Med 2020;382:1708-20.

18 Liu L, Yang Y, Gao Z, et al. Practice of diagnosis and management of acute respiratory distress syndrome in mainland China: a crosssectional study. J Thorac Dis 2018;10:5394-404.

19 Caputo ND, Strayer RJ, Levitan R. Early Self-Proning in awake, Non-intubated patients in the emergency department: a single
ED's experience during the COVID-19 pandemic. Acad Emerg Med 2020;27:375-8

20 Elharrar X, Trigui Y, Dols A-M, et al. Use of prone positioning in Nonintubated patients with COVID-19 and hypoxemic acute respiratory failure. JAMA 2020;323:2336.

21 Sartini C, Tresoldi M, Scarpellini P, et al. Respiratory parameters in patients with COVID-19 after using noninvasive ventilation in the prone position outside the intensive care unit. JAMA 2020;323:2338.

22 Zarantonello F, Andreatta G, Sella N, et al. Prone position and lung ventilation and perfusion matching in acute respiratory failure due to COVID-19. Am J Respir Crit Care Med 2020;202:278-9.

23 Xu Q, Wang T, Qin X, et al. Early awake prone position combined with high-flow nasal oxygen therapy in severe COVID-19: a case series. Crit Care 2020;24:250.

24 Tavernier E, Trinquart L, Giraudeau B. Finding alternatives to the dogma of power based sample size calculation: is a fixed sample size prospective Meta-Experiment a potential alternative? PLoS One 2016;11:e0158604

25 Li J, Pavlov I, Laffey JG, et al. Meta-trial of awake prone positioning with nasal high flow therapy: invitation to join a pandemic Collaborative research effort. J Crit Care 2020;60:140-2.

26 Rice TW, Wheeler AP, Bernard GR, et al. Comparison of the SpO2 $\mathrm{FIO} 2$ ratio and the $\mathrm{PaO} 2 / \mathrm{FIO} 2$ ratio in patients with acute lung injury or ARDS. Chest 2007;132:410-7.

27 Chen W, Janz DR, Shaver CM, et al. Clinical characteristics and outcomes are similar in ARDS diagnosed by oxygen saturation/Fio2 ratio compared with $\mathrm{Pao2} / \mathrm{Fio} 2$ ratio. Chest 2015;148:1477-83.

$28 \mathrm{Kim} \mathrm{K}$, Demets DL. Design and analysis of group sequential tests based on the type I error spending rate function. Biometrika 1987;74:149-54.

29 Jennison C, Turnbull BW. Group sequential methods with applications to clinical trials. CRC Press, 1999. 